\title{
Synthesis of 5-hydroxy-10-R-benzo[a]phenazine-12-oxides by cyclization of 2-arylamino-1,4-naphthoquinone-1-oximes under the action of nitrating mixture
}

\author{
C Leonid M. Gornostaev, ${ }^{1}{ }^{*}$ Elena V. Arnold, ${ }^{1}$ Daria S. Rudenko,${ }^{1+}$ Yulia G. Khalyavina, ${ }^{1}$ \\ Vladimir V. Chernyshev, ${ }^{2,3}$ Oleg A. Tyablikov, ${ }^{2}$ Sergey F. Dunaev, ${ }^{2}$ and Galina A. Stashina ${ }^{4}$ \\ ${ }^{1}$ Department of Biology, Chemistry and Ecology. V.P. Astaf'ev Krasnoyarsk State Pedagogical University, \\ Ady Lebedevoy, St. 89. Krasnoyarsk,660049.Russia.E-mail: gornostaev@kspu.ru \\ ${ }^{2}$ Department of General Chemistry M. V. Lomonosov Moscow State University. \\ Leninskie Gory St., 1, p.3. Moscow, 119991.Russia.E-mail:vladimir@struct.chem.msu.ru \\ ${ }^{3}$ A.N. Frumkin Institute of Physical Chemistry and Electrochemistry of the Russian Academy of Sciences. \\ Leninsky Ave., 31. Moscow, 119071. Russia. \\ ${ }^{4}$ N.D. Zelinsky Institute of Organic Chemistry, Russian Academy of Sciences. Leninsky Ave., 47. \\ Moscow, 119991.Russia.E-mail: galina_stashina@chemical-block.com
}

*Supervising author; ${ }^{+}$Corresponding author

Keywords: quinones, oximes, 5-hydroxy-10- $R$-benzo[ $[a]$ phenazine-12-oxides, cation-radical processes.

\begin{abstract}
The synthesis of polycyclic quinoid compounds, which exhibit a wide range of biological activity is one of the most promising and actively developing areas of the fine organic synthesis. Heterocyclic compounds including those that can be donors of nitrogen oxide NO occupy a special place among biologically active structures. These substances include a number of $N$-oxides, e.g., 1,2-diazet-1,2-dioxides, furoxanes and their benzo analogs, and $N, N^{\prime}$-pyrazole dioxides. The reason for the high biological activity of $N$-oxides of nitrogenous heterocycles, which cannot easily generate nitrogen oxide NO may be their oxidative properties. Thus, $N$-oxides of nitrogenous heterocycles are of interest due to their high biological activity.

We have developed an approach to the synthesis of 5-hydroxy-10- $R$-benzo[ $[a]$ phenazine-12-oxides that contain the $N$-oxide fragment, which makes these compounds promising for studying their biological activity. We have demonstrated that the treatment of 2-arylamino-1,4-naphthoquinones with hydroxylamine in ethanol at $50-60{ }^{\circ} \mathrm{C}$ leads to selective oximation at position 1 and the formation of 2-arylamino-1,4-naphthoquinone-1oximes. It has been shown that the interaction of 2-arylamino-1,4-naphthoquinone-1-oximes with the nitrating mixture in acetic acid results in the formation of 5-hydroxy-10- $R$-benzo[ $a]$ phenazine-12-oxides. Our work is a continuation of the study on the interactions of 2-arylamino-1,4-naphthoquinones and 4-arylamino-1,2naphthoquinones with nitrosylsulfuric acid in acetic acid and the interaction of 2-alkyl(benzyl)amino-1,4naphthoquinones with nitrosylsulfuric acid or nitrating mixture in acetic acid. The former reactions lead to the formation of 3-R-benzo[ $b]$ phenazine-6,11-dione-5-oxides and 9- $R$-benzo[ $a]$ phenazine-5,6-dion-7-oxides, respectively. The latter reaction leads to the formation of 2-alkyl(aryl)naphtho[2,1- $d][1,3]$ oxazole-4,5-dione4-oximes and 2-alkyl(aryl)-1-hydroxy-1 $H$-naphtho[2,3- $d$ ] imidazole-4,9-dione as main products.
\end{abstract}

\section{References}

[1] I.S. Severina, I.K. Ryaposova, L.B. Volodarsky et al. Biochem. Mol. Biol. Int. 1993. Vol.30. No.2. P.357-366.

[2] V.G. Granik, N.B. Grigoryev. Nitric oxide (NO). A new way to find drugs. Moscow: High school book. 2004. P.180. (russian)

[3] M. Feelisch, K. Schonafinge, E. Noak. Biochem. Pharmacol. 1992. Vol.44. P.1149-1157.

[4] C. Medana, G. Ermondi, R. Fruttero, A. Di Stilo, C. Ferretti, A. Gasco. J. Med. Chem. 1994. Vol.37. No.25. P.4412-4416.

[5] K. Schonafinger. Farmaco. 1999. Vol.54. P.316-319.

[6] M.B. e Silva T., T.V. Cerqueira N., W.D. Silva S. et al. Lett. in Organic Chemistry. 2011. Vol.8. No.10. P.752-762.

[7] K. Kobayashi, N. Oda, J. Sakakibara. Yakugaku Zasshi. 1983. Vol.103. P.165-172. 
SYNTHESIS OF 5-HYDROXY-10-R-BENZO[A]PHENAZINE-12-OXIDES BY CYCLIZATION..

[8] L.M. Gornostaev, V.B. Tsvetkov, A.A. Markova, T.I. Lavrikova, Y.G. Khalyavina, A.S. Kuznetsova, D.N. Kaluzhny, A.V. Shunayev, M.V. Tsvetkova, V.A. Glazunova, V.V. Chernyshev, A.A. Shtil. AntiCancer Agents in Medicinal Chemistry. 2017. Vol.17. No.13. P.1814-1823.

[9] RU Patent no. 2545 091. A.A. Shtil, V.A. Glazunova, T.I. Lavrikova, Yu.G. Khalyavina, L.M. Gornostaev. 2014. Byull. Izobret. 2015. No.9. (russian)

[10] S.P. Titova, A.K. Arinich, M.V. Gorelik. Russ. J. Org. Chem. 1986. Vol.22. P.1562-1564. (russian)

[11] E.A. Bocharova, L.M. Gornostaev, and N.V. Geets. Synthesis, structure of some nitrosoarenoles and 2nitrosodiphenilamines. Cyclization of 2-nitrosodiphenilamines into phenazine- $N$-oxides. Butlerov Communications. 2011. Vol.26. No.11. P.61-69. ROI: jbc-02/11-26-11-61

[12] L.M. Gornostaev, Yu.G. Khalyavina, T.I. Lavrikova, G.A. Stashina, S.I. Firgang, V.V. Chernyshev. Russ. Chem. Bull. (Int. Ed.). 2014. Vol.63. No.3. P.739-743. (russian)

[13] L.M. Gornostaev, E.V. Arnold, T.A. Lyashchenko. Chem. Heterocycl. Compd. 2014. Vol.49. P.18271830. (russian)

[14] L.M. Gornostaev, Yu.G. Khalyavina, T.I. Lavrikova, Yu.V. Gatilov, G.A. Stashina, S.I. Firgang. Russ. J. Org. Chem. 2014. Vol.50. No.12. P.1814-1820. (russian)

[15] L.M. Gornostaev, E.V. Nuretdinova, T.I. Lavrikova, Yu.G. Khalyavina, O.I. Fominykh and Yu.V. Gatilov. Russ. J. Org. Chem. 2019. Vol.55. No.5. P.608-614.

[16] L.M. Gornostaev, E.V. Nuretdinova, T.I. Lavrikova, Yu.G. Khalyavina, I.S. Kryukovskaya and Yu.V. Gatilov. Russ. J. Org. Chem. 2015. Vol.51. No.12. P.1733-1738.

[17] L.M. Gornostaev, M.V. Vigant, O.I. Kargina, A.S. Kuznetsova, Yu.G. Khalyavina, T.I. Lavrikova. Russ. J. Org. Chem. 2013. Vol.49. P.1354-1357.

[18] P.A. Belyakov, V.I. Kadentsev, A.O. Chizhov, N.G. Kolotyrkina, A.S. Shashkov, V.P. Ananikov. Mendeleev Commun. 2010. Vol.20. No.5. P.125.

[19] V.V. Chernyshev. Russ. Chem. Bull. (Int. Ed.). 2001. Vol.50. No.12. P.2273-2292.

[20] W.I.F. David, K. Shankland. Acta Crystallogr. Sect. A. 2008. Vol.64. P.52-64.

[21] K.D.M. Harris. Top. Curr. Chem. 2012. No.315. P.133-177.

[22] P.-E. Werner, L. Eriksson, M. Westdahl. J. Appl. Crystallogr. 1985. Vol.18. P.367-370.

[23] J.W. Visser. J. Appl. Crystallogr. 1969. Vol.2. P.89-95.

[24] V.B. Zlokazov. J. Appl. Crystallogr. 1992. Vol.25. P.69-72.

[25] V.B. Zlokazov. Comput. Phys. Commun. 1995. Vol.85. P.415-422.

[26] S.G. Zhukov, V.V. Chernyshev, E.V. Babaev, E.J. Sonneveld, H. Schenk. Z. Kristallogr. 2001. Vol.216. No.1. P.5-9.

[27] V.B. Zlokazov, V.V. Chernyshev. J. Appl. Crystallogr. 1992. Vol.25. P.447-451.

[28] L.M. Gornostaev, Yu.G. Khalyavina, A.S. Kuznetsova, O.I. Fominykh, D.A. Tropina, E.V. Murashova, I.A. Zamilatskov, V.V. Chernyshev. Russ. J. Org. Chem. 2016. Vol.52. No.1. P.80-87.

[29] S.-T. Zhuo et al. Organic \& Biomolecular Chemistry. 2013. Vol.11. No.24. P.3989-4005.

[30] H. Goldstein, P. Grandjean. Helv. chim. acta. 1943. Vol.43. No.9. P.468-475.

[31] A.L. Spek. Acta Crystallogr. Sect. D. 2009. Vol.65. No.2. P.148-155.

[32] C.R. Groom, F.H. Allen. Angew. Chem. Int. Ed. 2014. Vol.53. P.662-671.

[33] Z. Wróbel, K. Stachowska, A. Kwast. Eur. J. Org. Chem. 2014. Vol.34. P.7721-7725.

[34] G. Foyer. Chemistry of nitro and nitroso groups. Vol.1. Moscow: Mir. 1972. P.108. (russian)

[35] R.K. Norris, S. Sternhell. Aust. J. Chem. 1972. Vol.25. P.2621-2629. 